\title{
Téoros
}

Revue de recherche en tourisme

\section{Le rôle des informations touristiques dans la médiatisation du territoire de visite}

\author{
Le cas d'un voyage semi-organisé à Paris
}

\section{Gwendal Simon}

Volume 31, numéro 2, 2012

URI : https://id.erudit.org/iderudit/1020770ar

DOI : https://doi.org/10.7202/1020770ar

Aller au sommaire du numéro

Éditeur(s)

Université du Québec à Montréal

ISSN

0712-8657 (imprimé)

1923-2705 (numérique)

Découvrir la revue

Citer cet article

Simon, G. (2012). Le rôle des informations touristiques dans la médiatisation du territoire de visite : le cas d'un voyage semi-organisé à Paris. Téoros, 31(2), 42-50. https://doi.org/10.7202/1020770ar
Résumé de l'article

Les représentations catégorisent aisément le tourisme de groupe comme une expérience « institutionnalisée » référant à un tourisme de masse où les pratiques seraient à la fois encadrées et largement dirigées vers les objets et des services identifiés comme touristiques. Ainsi, la voie serait ouverte pour une expérience peu authentique. La compacité et l'univocité de tels voyages sont aujourd'hui très affaiblies par la différenciation croissante des offres proposées et la prise en compte de la capacité réflexive des touristes au cours de leur voyage. Celle-ci se révèle inégale selon l'acculturation au territoire de visite. C'est l'hypothèse principale de cet article qui, à travers l'exemple de la diffusion des informations opérées par un voyagiste à Paris et de leur réception par les touristes étrangers, souligne combien la médiatisation d'un territoire dans le cadre d'un voyagiste résulte d'une coconstruction entre les professionnels du voyage et des touristes eux-mêmes.
Ce document est protégé par la loi sur le droit d'auteur. L'utilisation des services d’Érudit (y compris la reproduction) est assujettie à sa politique d'utilisation que vous pouvez consulter en ligne.

https://apropos.erudit.org/fr/usagers/politique-dutilisation/ 


\title{
Le rôle des informations touristiques dans la médiatisation du territoire de visite Le cas d'un voyage semi-organisé à Paris
}

\author{
Gwendal SIMON \\ Maître de conférences \\ Université Paris Est Marne-La-Vallée \\ Laboratoire Ville Mobilité Transport (Ecole des Ponts Paris Tech, Université Paris Est, IFSTTAR) \\ gwendal.simon@enpc.fr
}

\begin{abstract}
RÉSUMÉ: Les représentations catégorisent aisément le tourisme de groupe comme une expérience «institutionnalisée » référant à un tourisme de masse où les pratiques seraient à la fois encadrées et largement dirigées vers les objets et des services identifiés comme touristiques. Ainsi, la voie serait ouverte pour une expérience peu authentique. La compacité et l'univocité de tels voyages sont aujourd'hui très affaiblies par la différenciation croissante des offres proposées et la prise en compte de la capacité réflexive des touristes au cours de leur voyage. Celle-ci se révèle inégale selon l'acculturation au territoire de visite. C'est l'hypothèse principale de cet article qui, à travers l'exemple de la diffusion des informations opérées par un voyagiste à Paris et de leur réception par les touristes étrangers, souligne combien la médiatisation d'un territoire dans le cadre d'un voyagiste résulte d'une coconstruction entre les professionnels du voyage et des touristes eux-mêmes.
\end{abstract}

Mots-clés: Paris, voyagiste, information, encadrement, expérience.

L'histoire du tourisme de groupe est relativement récente et émerge au XIX ${ }^{e}$ siècle bien que certains observateurs aient pu retrouver, dans la Rome antique, la trace de médiateurs qui participaient à faciliter le voyage. Malgré ces formes premières, Lanquar (1995) note que cette histoire ne commence vraiment que vers 1800, lorsque des amateurs de bateaux à vapeur, en Grande-Bretagne, offrirent des services réguliers le long des côtes et engagèrent des agents généraux maritimes. Thomas Cook est généralement présenté comme un des pionniers du voyage "organisé » en étendant l'encadrement à l'ensemble du voyage, et non à une simple logistique de transport pour les touristes (Enzensberger, 1962; Boyer, 1999). Ces évolutions sont la résultante d'une inventivité commerciale et du progrès technique des moyens de transport, supports logistiques nécessaires au déplacement collectif. L'autocar et l'avion vont grandement faciliter l'extension des offres commerciales des agences de voyage en ouvrant des destinations nouvelles et en favorisant les circuits. C'est alors une organisation qui n'aura de cesse de s'étendre avec la démocratisation du tourisme, dont la particularité essentielle consiste à introduire une logique de services dans l'univers du voyage.
Cohen (1972) a catégorisé le tourisme de groupe comme une expérience "institutionnalisée» renvoyant à un tourisme de masse plus ou moins organisé, où les pratiques sont largement dirigées vers les objets et des services identifiés comme touristiques et tout ou partiellement encadrées. La compacité d'une telle expérience de voyage doit cependant être relativisée sur un marché devenu complexe (Veneto, 2007 ) et où, en conséquence, les voyagistes doivent adapter leur offre en l'individualisant (Bauer, 2007). La dimension institutionnalisée de ces voyages est donc moins évidente que par le passé, même si l'organisation et la sécurisation des touristes constituent toujours des éléments clés. Ceux-ci sont lisibles sur un plan à la fois matériel (par des services pratiques contribuant au confort du voyage) et culturel (par la diffusion d'informations et de conseils). La diffusion de l'information par des personnes qualifiées a toujours été structurante des voyages guidés (Holloway, 1981) et constitue un facteur stratégique dans la représentation de la destination, influençant la qualité de l'expérience touristique (Dalhes, 2002). Plusieurs études ont montré l'importance des guides dans la sphère du tourisme culturel (Bowman, 1992) où leurs activités consistent à sélectionner et interpréter les éléments 
signifiants du territoire de visite pour les transmettre aux touristes (Schmidt, 1979). Une de leurs tâches principales est de traduire "the strangeness of a foreign culture into a cultural idiom familiar to the visitor» (Cohen, 1985 : 15), suscitant le sentiment d'un contact cutlurel (Bowman, 1992).

Le rôle des guides est de transmettre des informations, d'offrir des explications et de développer des récits sur le territoire de visite : "the guide is entrusted with the public relations missions to encapsulate the essence of a place to be a window into a site, a region, or country» (Pond, 1993). Les guides, comme les agents réceptifs, ont ainsi un rôle essentiel de médiation et sont souvent dépeints comme "passeurs», notamment entre les touristes et le territoire local. L'objet que nous investiguons concerne la circulation des informations entre le voyagiste - par l'intermédiaire des agents réceptifs et des guides-interprètes - et les touristes. Nous faisons l'hypothèse que les informations et conseils touristiques transmis par les voyagistes sont diversement assimilés selon l'acculturation au territoire de visite : ceux qui découvrent pour la première fois le territoire s'engageraient aisément dans les services proposés par le voyagiste comparativement aux plus confirmés qui tendraient à s'en affranchir. Pour démontrer cette hypothèse, nous interrogerons le cadre d'analyse du tourisme de groupe et des voyages organisés pour comprendre ensuite comment s'incarne concrètement le rapport à l'encadrement dans une configuration précise de voyage. Nous analyserons une configuration de voyage semi-organisé à Paris qui est proposé par un voyagiste finlandais. L'objectif est de comprendre la logique de diffusion des informations (sur quoi portent-elles?) et leurs réceptions par les voyageurs (qu'en font-ils?). Cette confrontation entre l'offre de conseils et son accueil permet de saisir les significations individuelles attribuées aux informations fournies.

\section{Le positionnement et la méthodologie de recherche}

Processus de construction et de sélection, la description sociologique est indissociable d'un regard théorique et interprétatif qui la guide et la rend «utile» (Lahire, 2005). C'est le modèle d'un acteur doué d'une réflexivité partielle, activée dans le cadre du voyage touristique qui forme notre référence théorique. Ce positionnement donne à l'acteur une capacité d'action stratégique. On part de l'hypothèse que les touristes possèdent des capacités d'action et de réflexion dans le cadre de leur voyage. Un ensemble d'éléments militent pour considérer les touristes comme acteurs de leurs pratiques : le voyage se réfléchit en amont de sa manifestation physique, notamment lorsqu'il est régi par le loisir, c'est-à-dire par un temps dévolu à soi (Bellefleur, 2002). La réflexion opère également par la distance au quotidien que réalise le voyage touristique dans une certaine rupture avec le cours régulier des choses : des choix et des arbitrages sont à faire, un projet se dessine (Urbain, 2003). Néanmoins, ce n'est pas dire que les touristes ont une rationalité globale et complète, détachée de tout environnement social et de tout contexte d'action. L'intentionnalité est ici rapportée au choix d'un projet de voyage organisé par un professionnel qui fixe des normes et des valeurs. Ainsi, la rationalité n'est ni totale et acontextuelle, car elle est accolée aux formes de voyages et à des propriétés sociales correspondantes (un certain âge, des niveaux de revenu, etc.) et peut être questionnée sur cette base. Devant les conseils du voyagiste, dans quelle mesure les touristes les combinent et les hiérarchisent ou produisent des arbitrages? L'approche compréhensive — le sens subjectif que le touriste donne aux logiques animant ses actions - apparaît comme la méthode idoine en réponse à ce cadre d'analyse. Avec Weber (1921), la théorisation d'une perspective compréhensive du monde social a participé à asseoir la légitimité des représentations subjectives comme modèle explicatif de l'action, même s'il n'a pas abordé tel quel les questions méthodologiques, les concevant essentiellement d'un point de vue épistémologique (Colliot-Thélène, 2006). Max Weber (1921 : 4), en parlant de "sens subjectif», a préfiguré l'analyse de ce qui est aujourd'hui dénommé, de façon disparate, comme le "vécu» des acteurs. Cette compréhension supposant le recueil du discours, l'entretien se révèle une des techniques les plus satisfaisantes pour qu'il se construise et se développe.

La méthodologie développée pour l'étude de cette configuration de voyage est celle d'une enquête qualitative combinant des entretiens semi-directifs avec différents acteurs impliqués dans le voyage organisé et des séquences d'observation au sein de ce dernier. Les entretiens ont visé trois types d'acteurs : le premier concerne les guides-interprètes travaillant dans la métropole parisienne $(n=11)$ qui ont été sollicités pour décrire les formes du tourisme organisé dans les métroppoles dont Paris. Les deux autres sont directement orientés vers le voyagiste lui-même : ce sont à la fois les «touristes» en déplacement avec celui-ci $(n=29)$, interrogés sur leur relations à l'encadrement et aux informations transmises par ce dernier, et enfin les agents réceptifs responsables de la destination $(n=3)$. Ceux-ci ont le rôle de guides et d'encadrants et ont été questionnés sur leur activité et le contenu des informations transmises aux touristes. Les formes et les impacts de la médiation opérées par les informations des agents réceptifs sur les touristes sont ici reconstitués, par les entretiens, dans le cadre d'un récit sur l'expérience que vivent les touristes (comment racontent-ils leurs rapports aux prescriptions proposées). Ces phases d'entretien ont été complétées par des séquences d'observation non participantes dans les espacestemps où les encadrants interagissent avec les touristes : réunion d'information, visites guidées, tour de la ville en autocar. Ces observations offrent une connaissance des manières de faire qui ne sont pas verbalisées lors des discours.

\section{Le groupe, une notion à déconstruire}

Le balancement entre des logiques d'encadrement du collectif et des dynamiques d'individualisation questionne les catégorisations du tourisme de groupe. L'encadrement n'est pas un tout qui s'impose au touriste, et celui-ci n'est pas un simple agent passif de son voyage ( $\mathrm{Li}, 2000)$. Les voyages organisés par des professionnels ne se résument pas à des formules «tout compris» dans le cadre d'une planification maximale délestant les touristes de toutes questions pratiques et, plus largement, de réflexivité concernant leur expérience de voyage (Wickens, 2002). 
Pourtant, les approches développées dans les années 1960 et 1970 présentent une figure relativement homogène du touriste. Que l'expérience soit inventée et préfabriquée par le tourisme de masse (Boorstin, 1963) ou une recherche d'expériences authentiques (MacCannell, 1976), les besoins et les désirs des touristes furent appréhendés comme relativement constants et peu sensibles à la différenciation sociale comme à la diversité des contextes. Parmis les premiers, Cohen (1972, 1973) va conceptualiser l'expérience touristique en s'appuyant sur la différenciation des motivations individuelles, proposant une typologie sur la base d'un degré d' «institutionnalisation » entre un tourisme de masse organisé et un tourisme plus fortement individualisé. Il distingue le tourisme institutionnalisé (institutionalized tourism) de son opposé (non institutionali$z e d)$. La forme institutionnalisée, c'est-à-dire le tourisme de masse organisé, se partage entre ceux qui voyagent en groupe (organized group) et les autres (individual mass tourists). Ceux-ci sont globalement pris en charge par un professionnel du voyage. Les premiers restent largement confinés dans un environnement peu ouvert sur l'extérieur au sein des structures prévues par le voyagiste, comme le tout inclus, par exemple, où l'encadrement et le degré de sécurisation viennent pallier la rupture avec le quotidien. Les seconds (les «individuels») en constituent une version plus souple : le degré de planification est plus faible, il est possible de maîtriser le temps et l'itinéraire et, enfin, l'affiliation au groupe n'est pas permanente.

Dans un contexte marchand qui a profondément changé (désintermédiation, différenciation de l'offre), cette distinction a perdu sa cohérence. D'un point de vue analytique, deux éléments peuvent être questionnés : la notion de groupe et celle d'encadrement du voyage par des intermédiaires spécialisés. Ils soulèvent deux questions : que recouvre l'expression de «tourisme de groupe» et quel peut-être son champ ou ses champs d'application, d'une part; et que signifie également voyager par l'encadrement d'un intermédiaire spécialisé, d'autre part? Pour y répondre, nous nous basons sur les informations recueillies lors des entretiens avec les guides-interprètes qui permettent de comprendre les formes multiples du tourisme de groupe.

Le groupe de touristes ne réfere pas à une signification unique synonyme d'une grégarité complète sur l'ensemble du voyage ( $\mathrm{Li}, 2000)$. Différents types de groupes sont possibles : il y a ceux que les personnels des agences de voyage nomment couramment les "groupes constitués", où le groupe se trouve préalablement formé avant même le départ. Ce peut être une classe de scolaires, un groupe de gens d'affaires invités par la même entreprise qui se déplace pour assister à un séminaire, etc. Le groupe est déjà « constitué », ses membres se connaissent plus ou moins et se calent sur une offre ou la composent selon leurs choix et leurs moyens. On parle également des groupes comme des «individuels regroupés ", où le groupe n'existe pas en tant que tel avant le voyage, les touristes ne se connaissant généralement pas. L'arrivée à l'aéroport est généralement le moment du «regroupement» pour l'ensemble de celui-ci ou pour certaines visites, voire pas du tout si l'on ne choisit pas les prestations proposées. Dans tous les cas, le groupe se reforme ici lors du départ vers l'aéroport. Le regroupement collectif peut aussi n'être que très provisoire, le temps d'une visite guidée, sur place, et uniquement le temps de cette visite, le guide réunissant le groupe dans un temps et un lieu bien défini. Ainsi les modalités des voyages « collectifs» sont diverses, appelant finalement des catégories hydrides telles que semi-groupe, individuels regroupés, etc. Ce type de segmentation pose des difficultés aux spécialistes du marketing qui furent habitués à un marché compact, en partie dominé par les seniors alors que l'âge n'est, aujourd'hui, plus un critère discriminant sur un marché qui se complexifie (Veneto, 2007). C'est pourquoi, dans ce contexte d'évolution, les voyagistes doivent adapter leur offre de tourisme de groupe en cassant le côté standardisé des produits (grands hôtels, grands autocars, grande salle pour la restauration) et en individualisant et en personnalisant l'offre. Ils s'agit d'assouplir l'homogénéité du groupe, en somme. Si cela ne traduit pas nécessairement une «désinstitutionnalisation» en bloc des pratiques, il y a différents degrés dans l'affiliation aux formes "institutionnalisées » parce qu'il y a différentes formes de collectifs de touristes. Différentes relations à l'«institutionnalisation» sont ainsi à penser.

$\mathrm{Au}$ niveau de l'encadrement par un voyagiste ou de ses services afférents (guide, etc.), les représentations tendent à concevoir l'encadrement comme un bloc homogène et le touriste comme un suiveur. Pourtant, de multiples formes d'encadrement existent, certaines sont très serrées, où l'emploi du temps est chargé, pleinement défini alors que d'autres offrent d'importants moments de liberté. L'encadrement peut-être global et latent, c'est-à-dire qu'à tout moment, alors que les plages horaires ne sont pas planifiées, un guide ou un responsable est disponible pour aider et conseiller. À contrario, un touriste "individuel», voyageant seul et sans encadrement particulier, peut faire appel à un guide pour une heure, une matinée, une journée, trois jours, une semaine. Ainsi, les possibilités de recourir à ce type d'intermédiaire sont nombreuses et la relation au voyagiste est plurielle. L'encadrement n'engage donc pas de rapport strictement univoque pour les touristes. C'est davantage dans une mécanique plus souple — située dans des offres commerciales, des contextes de voyage, des interactions des touristes aux encadrants - qu'il est nécessaire de replacer cette relation.

\section{L'observation d'un voyage semi-organisé à Paris Données générales}

Paris constitue une force d'attraction majeure en termes d'images et de flux touristiques, et bénéficie d'un pouvoir d'évocation considérable en stimulant les représentations et les récits. Depuis longtemps parmi les premières destinations touristiques dans le monde, elle a attiré 28 millions de touristes en 2011 (OTCP, 2012). Paris s'est progressivement constitué, au moins depuis le XIX ${ }^{\mathrm{e}}$ siècle, en s'organisant principalement autour d'un espace central historique (Fagnoni et Aymard, 2002).

Le développement relativement continu des flux touristiques vers Paris repose, en partie, sur une croissance du tourisme de groupe (APUR, 2001). Cette enquête estime qu'environ 27,5 \% du nombre de séjours réalisés en Île-deFrance sont organisés par un professionnel du tourisme, dont la part la plus importante est le collectif formé par les voyagistes $(65 \%)$, largement devant les groupes scolaires (12\%), les 
groupes de retraités $(7 \%)$ et les groupes d'entreprise $(6 \%)$ (APUR, 2001). Les touristes étrangers y sont largement majoritaires (environ $82 \%$ ), car nombre de touristes français qui voyagent à Paris sont hébergés par des tiers ou s'organisent seuls (APUR, 2001). Si le poids des voyages organisés diffère selon l'origine des touristes (majoritairement des pays développés), plus la provenance des touristes est lointaine, plus le recours à un intermédiaire pour réserver son voyage est fréquent (APUR, 2001). Au final, un quart des touristes à Paris voyagent par le biais d'un intermédiaire et, pour l'essentiel, dans le cadre d'un groupe. Les circuits restent globalement marqués par des courtes durées et des polarisations sur les lieux emblématiques du tourisme, dessinant un tableau assez standardisé même si des marchés moins connus et alternatifs aux parcours classiques existent (Holm, 2003; Sallet-Lavorel, 2003). La configuration de voyage étudiée s'inscrit globalement dans ce panorama.

Pour la présente étude, le voyagiste choisi est finlandais : il s'agit d'une filiale de Finnair, la plus grande compagnie aérienne finlandaise. Transportant environ 300000 personnes annuellement, il est considéré comme un des voyagistes les plus importants du pays parmi un ensemble de 4 à 5 firmes dominant le marché. Paris est, pour ce voyagiste, la seconde ville la plus prisée en Europe, après Rome, bien qu'elle représente une part très mineure dans l'ensemble de destinations proposées dans le monde, d'après un des agents réceptifs interrogés. Les touristes ont à choisir une durée qui est modulable en fonction des formules "week-end» (trois jours), "grand weekend» (cinq jours) et «semaine» (sept jours). La clientèle tend globalement à se répartir équitablement sur l'ensemble de ces durées mais avec des variations selon les périodes de l'année. Les hôtels sont situés dans le quartier des Grands Boulevards, à proximité de l'Opéra et des Grands Magasins, soit dans l'hypercentre parisien. Classés "trois étoiles», ils correspondent à un niveau de bon confort qui est relativement répandu à Paris. Les classes d'âge se situent sur un intervalle qui va approximativement de 40 à 60 ans. Le niveau de vie correspond à des classes moyennes, voire moyennes supérieures. En effet, les touristes que nous avons rencontrés — dans le cadre de brefs échanges ou d'entretiens formels - occupent des postes de chauffeur de tramway ou de chauffeur de train, sont instituteurs ou institutrices, enseignants ou enseignantes de lycées, ingénieurs ou ingénieures, cadres commerciaux, sous-officier de l'armée, retraités ou femmes au foyer, etc. Si l'on prend les diverses professions typiques classées selon le salaire mensuel net médian en euros disponibles dans l'ouvrage de L. Chauvel (2006) sur les classes moyennes françaises (mais dont on peut supposer que les classes moyennes finlandaises ne diffèrent pas tellement si l'on regarde les différents indicateurs statistiques fournis par l'OCDE (Kharas, 2010)), on retrouve, dans notre échantillon, des professions similaires. En effet, elles ressortent des classes moyennes intermédiaires (professions administratives dans des collectivités, les agents de police, instituteur, institutrice, pompier, pompière) et des classes moyennes supérieures (ingénieur, cadre administratif ou financier, personnel de direction, officier de l'armée, etc.). Ce ciblage reste partiel mais offre un aperçu cohérent sur l'élément tarifaire. La répartition par genre est légèrement déséquilibrée au profit des femmes. Concernant le nombre de personnes composant le voyage, les données varient selon les saisons, surtout l'été où les familles sont nombreuses. En hiver, davantage de personnes viennent à Paris pour des raisons qui sont professionnelles (salons, foires, etc.) ou pour des événements spécifiques (par exemple, suivre des courses hippiques, des compétitions sportives, etc.). Le printemps est davantage une période charnière où se mélangent ceux qui voyagent avec des intérêts clairement touristiques et d'autres qui sont plus hybrides (associant des activités professionnelle et touristique).

\section{Niveau d'encadrement et programme proposé}

En termes d'organisation et de contenu du voyage, le voyagiste offre un haut niveau de services, mais dont une partie est optionnelle, et un contenu qui propose la visite des hauts lieux du tourisme à Paris (dont la décision appartient au touriste). Un ensemble de temps et de documents spécifiques sont dévolus à l'accompagnement physique et cognitif des touristes, notamment lors du transfert entre l'aéroport et l'hôtel et pendant une réunion d'information le jour de l'arrivée. Ils reçoivent une brochure informative et ils peuvent consulter, librement et à tout moment, un classeur disponible dans chaque hôtel avec de nombreuses indications sur la ville. L'ensemble est chapeauté par la présence de l'agent réceptif de façon presque continue par téléphone et régulièrement par les permanences que cet employé effectue trois fois par semaine dans les différents hôtels. Outre cet encadrement potentiellement fort et mobilisable en fonction des besoins de chacun, le programme de visite est classique, renvoyant aux dimensions référentielles du patrimoine parisien : paysager, historique, architectural.

Les excursions sont proposées en option et libre, partiellement ou en totalité, selon son appétence, son budget et la durée du voyage. La dimension classique est lisible dans les visites à Versailles et au musée du Louvre, qui sont tous deux des hauts lieux du tourisme culturel. Le «tour de ville» offre un circuit qui serpente dans l'espace touristique central identifié à Paris (Duhamel et Knafou, 2007) et qui fonctionne comme une présentation d'ordre général de la ville. Les circuits proposés suivent toujours les mêmes arrondissements, traversant ainsi Paris au travers de ses éléments les plus visibles.

L'homogénéité de ces touristes est déterminée par la structuration minimale obligatoire fournie par le voyagiste : un vol aller-retour pour Paris, un hébergement parmi un ensemble de plusieurs hôtels de gamme moyenne, le transfert aller et retour entre l'aéroport et l'hôtel, un groupe provisoirement constitué à certains moments, la présence de concitoyens à l'hôtel. Cette homogénéité est aussi déterminée par un cadre temporel assez restreint où les deux tiers des touristes voyagent cinq jours ou moins. Elle est également structurée par un cadre budgétaire défini à minima (même aux tarifs les plus bas, il appelle un budget minimum de 350 euros pour trois jours) où l'âge et le niveau social globalement assez élevés postulent, à priori, un pouvoir d'achat qui, sans être extensif, n'est pas réduit. Le niveau d'encadrement élevé mais potentiel joue à la manière d'une sécurisation mobilisée selon les besoins. D'ailleurs, il y a là un niveau minimal moyen qui figure un socle commun. Néanmoins, l'aspect potentiel des services et du programme proposé permet de combiner plus 
individuellement, selon ses moyens financiers, ses désirs ou ceux des autres, une configuration de voyage plus personnelle et qui est plus ou moins en mesure de se détacher de l'assise initiale. Il y a ainsi une labilité possible dans l'engagement des touristes face à l'encadrement fourni.

\section{La diffusion et la réception des informations L'articulation des discours émetteurs et récepteurs}

Il est difficile d'articuler pleinement le discours émetteur et les réceptions qu'en font les touristes. Comment, en effet, arriver à appréhender les effets des discours "émetteurs» sur une «multiplicité» de voyageurs? La complication réside dans la dissymétrie entre le caractère général et parfois monolithique du discours proféré par les agents réceptifs (qui est répété, plusieurs fois par semaine depuis plusieurs années) et les touristes qui l'intériorisent inégalement. Il n'est donc pas aisé de tracer les conseils des encadrants jusque dans les pratiques de ceux qui, à priori, les ont écoutés. La reconstitution de ce parcours se perd parfois dans les méandres des pratiques concrètes dont les touristes ne perçoivent plus, ou mal, le caractère opératoire quand il s'agit de les remémorer lors de l'entretien avec les touristes. Celui-ci se faisant à la fin du séjour, l'efficace de tels conseils - importants lors de l'arrivée, car le sentiment de coupure est le plus net - est moins palpable dans l'esprit de certains enquêtés. S'il l'on peut parler de progressivité dans l'apprentissage du territoire de visite (à travers la sensation d'un engagement moins hésitant dans l'action et d'une certaine autonomie au fur et à mesure du contact avec la ville), le regard rétrospectif des touristes enquêtés sur ses pratiques oublie parfois ce qu'elles doivent aux formes d'encadrement. En effet, le suivi s'altère et ce sont sous les impressions de l'autoréalisation qu'il faut alors chercher. Cependant, cette méthode ne fonctionne pas toujours, car les enquêtés peuvent actionner, sur un mode plus ou moins conscient, des stratégies de défense de l'autonomie et de l'action individuelle qui jouent comme des barrières pas toujours bien perméables ou contournables (on touche là à une des limites des recueils de données par l'entretien). Néanmoins, ces limites ponctuelles n'obèrent pas la lecture d'«effets» dans le discours des enquêtés sur leurs pratiques : effets performatifs du discours des encadrants mais aussi représentations différenciées de ceux-ci par les touristes.

\section{Le «transfert»: de l'aéroport au cœur de la ville}

Ce temps d'accompagnement des touristes entre l'aéroport et l'hôtel, supervisé par un encadrant, permet de fournir un premier lot d'informations outre la prise en charge du déplacement. Comparativement au temps du retour, plus calme et apaisé car le voyage est "derrière soi», le temps de l'aller mobilise un discours spécifique qui présente l'ensemble des modalités pratiques d' «accès» à la destination, tant géographiques que fonctionnelles. Si l'on distille quelques éléments historiques en fournissant des repères selon les sites adjacents au fur et à mesure de l'arrivée dans la ville, le discours se concentre sur les aspects pratiques du voyage. Dans le cas de Paris, il s'agit, d'une part, du détail des conditions d'arrivée à l'hôtel puis, d'autre part, du développement des appuis possibles pour voyager avec fluidité. Il est question notamment de mettre à jour les mobilités adéquates pour bouger (métro, taxi), les conseils visant la sécurisation de soi et de ses affaires. Ce temps du transfert consiste en une présentation assez hétéroclite des modalités concrètes pour vivre la ville. C'est la pose, "à l'avance», de certains repères qui structurent la représentation d'un espace qui est, géographiquement, encore à connaître, au moins pour certains.

L'information issue du «transfert» n'empêche néanmoins pas l'ambivalence, car, si elle se veut un socle théorique pour l'action dès l'arrivée, sa réception ne se traduit pas toujours par une intériorisation complète par ces touristes des données fournies sur la ville. Si le temps du transfert est un temps «obligé» et commun aux clients, ils sont libres d'écouter attentivement ce discours, de mobiliser une attention plus sélective ou aléatoire, voire de s'y soustraire (ne pas écouter). L'attention n'est pas homogène et l'information est diversement reçue. La difficulté tient dans la volonté du voyagiste de pallier les effets de l'arrivée par un encadrement estimé adéquat alors même que ce temps est marqué par une dispersion de l'attention vers l'environnement extérieur. On parle d'histoire, d'hébergement, de transports, de bonnes pratiques, on distribue et commente une brochure, on détaille les excursions possibles. L'information, pour être reçue, demande une concentration des touristes qui, justement parce qu'ils «arrivent ", ne sont pas toujours disposés à tendre l'écoute dans une seule et même direction. En conséquence, l'information ne passe pas toujours bien. Ce n'est pas seulement parce qu'elle est diffusée lors d'un trajet en car dans lequel les interférences sont nombreuses mais aussi parce qu'elle est dense. Un touriste explique :

On oublie des choses, elle [la guide] dit tellement de choses... D'abord elle parle de l'histoire et puis du métro, et puis à nouveau l'histoire, donc c'est... ça m'a plus rendu plus confus qu'autre chose, donc euh... je pense que j'ai entendu sur les transports ce que je devais entendre, c'est-à-dire que je pouvais utiliser le métro et les taxis mais, après, j'ai un peu oublié...

(Paavo, 43 ans, 2006 : communications personnelles).

La confusion est aussi parfois redoublée parce que la réunion d'information suit de près ce temps de transfert et provoque l'effet d'un doublon. La réception résulte aussi de l'affaiblissement du discours pour ceux qui ont déjà à l'esprit ces données du fait de plusieurs écoutes antérieures ou parce que certains enquêtés ont l'habitude de voyager dans des conditions similaires. Dans ce cas, l'attention est clairement flottante et se distribue entre l'observation extérieure, les discussions éventuelles et l'écoute partielle:« [La guide] était plus concentrée sur... enfin nous étions au fond du bus donc on ne la voyait pas bien, on n'entendait pas trop, mais je suppose qu'elle organisait les choses donc... mais elle a parlé de... elle n'a pas vraiment parlé de ce qu'il y avait autour de nous, je crois qu'elle a parlé de comment gérer l'hôtel et de l'arrivée...» (Risto, 56 ans, 2006 : communications personnelles).

\section{À l'arrivée : la réunion d'information}

Une rencontre d'information est généralement offerte aux visiteurs dès leur arrivée, soit le premier matin, s'ils arrivent la veille, ou le premier après-midi, s'ils sont arrivés en matinée. 
Ce temps est une reprise plus complète des informations communiquées lors du transfert. L'effet de redoublement est néanmoins pallié par un environnement matériel et cognitif plus stabilisé qui limite les interférences et favorise les interactions entre l'encadrant et les touristes. C'est à la fois un moment fondateur en tant qu'il marque l'arrivée à Paris et le partage collectif de cette expérience. La réunion commence par la présentation des différents modes et de leur fonctionnement sur la base du plan des transports en commun préalablement fourni lors du transfert, outil nécessaire à une représentation théorique du réseau. L'efficacité du métro est soulignée et les agents réceptifs partagent l'idée d'un apprentissage assez «intuitif » qui appelle généralement moins le recours in situ à des médiations extérieures que le bus. Ils jugent que si l'acculturation au métro est progressive en se réalisant au prix d'hésitations et de quelques erreurs, elle se traduit rarement par des désorientations problématiques. Toutefois, si l'apprentissage se fait sans heurt notable, c'est qu'il y a, en arrière-fond, la transmission théorique de savoirs pratiques sur cet univers qui permet de construire abstraitement un itinéraire, d'avoir des éléments pour choisir adéquatement les différents tarifs et des conseils pour s'accorder avec l'environnement sociotechnique (les guichets et les distributeurs de titres, etc.).

Les informations sur la ville sont triplement détaillées : ce sont à la fois les possibilités de "promenade», de visites culturelles, des modalités d'être ou de faire dans les cafés et les restaurants qui sont conseillées et étayées de détails pratiques. «Les informations sont utiles... on a eu des informations que nous n'avions pas au début, en venant [lors du transfert], sur des endroits que nous ne connaissions pas, ou sur des choses dont nous avions entendu parler mais que nous avions oubliées [...] comme les cimetières. J'avais oublié qu'il y avait la tombe de Jim Morrison et toutes ces célébrités» (Noora, 55 ans, 2006 : communications personnelles). Le discours sur l'espace possible de circulation piétonne de la ville est illustré à partir d'un itinéraire théorique qui est proposé et "réalisé » par le plan que les touristes ont avec eux lors de la réunion. Les différences culturelles et les manières de faire localisées impliquent de spécifier les décalages afin de limiter les désagréments pour ne conserver que la curiosité de pratiques inhabituelles dont on a été préalablement averti. Ainsi, la réunion d'information, sous l'angle du discours informatif, «déplie» ici en quelque sorte la ville sous trois angles qui s'entremêlent : paysager, culturel et marchand. Un des aspects de ce temps propre à la diffusion d'information est de constituer un cadre propice aux interactions.

\section{Des interactions stabilisées}

La réunion d'information tenue en début de séjour est un temps partiellement convivial qui se déroule concrètement dans le calme et le cadre presque clos de la salle qui suscite une dynamique d'échanges. Le discours produit soulève un ensemble de questions auquel le guide essaie généralement de répondre une fois son propos terminé : "J'écoute quels sont les problèmes [...] Par exemple "la tour Eiffel, combien ça coûte, comment monter?” Ça, c'est une question qu'ils posent souvent, "combien de temps faut-il attendre pour arriver au sommet de la tour Eiffel ?"» (Pauliina, agent réceptif, 2006 : communications personnelles). Il y a un ensemble de problèmes qui sont abordés et résolus abstraitement, mais dont il faudra nécessairement les tester pour en négocier la maîtrise pratique, notamment la relation avec l'environnement du métro. Concernant les informations sur la ville, les questions sont nombreuses et variées, touchant les lieux possibles de magasinage, les adresses d'un restaurant ou d'une supérette de proximité ou encore les modalités pour se rendre à Disneyland.

La dimension stabilisée des interactions est encore lisible à partir des documents qui sont fournis aux touristes à travers la brochure informative et le plan des réseaux de transports qui servent de support aux discours prononcés par les encadrants. La mémoire pratique issue des routines quotidiennes ne pouvant ici être sollicitée, elle est objectivée par des annotations, des soulignements, des griffonnements, etc. En effet, nombreux sont les plans de transport dont des stations sont entourées ou cochées, fréquentes aussi sont les brochures informatives qui sont complétées par des notes : "J'ai noté des choses à voir, vous savez, je les ai marquées d'une croix dessus... les numéros de bus, les rues où faire du shopping et $[. .$.$] combien coûte le ticket-journée, vous savez, le ticket$ de métro... des choses comme ça, pour me rappeler» (Heidi, 40 ans, 2006 : communication personnelles); "J'ai juste fait quelques croix sur la carte et les stations de métro [...] c'est la station où on doit se rendre pour aller à Disneyland [...] je n'aurais pas à rechercher» (Teuvo, 44 ans, 2006 : communications personnelles). Ces micros pratiques scripturales suppléent la mémoire individuelle qui n'incorporent pas aisément la somme des informations fournies. En conséquence, ces actions permettent de se rassurer et d'organiser les futurs mouvements par une représentation sommaire du territoire : quelques grandes aménités touristiques et des zones marchandes servent de repères, des stations de métro émergent et offrent une première structuration spatiale.

Une autre manière de considérer la réception consiste à la situer plus largement non plus seulement au niveau des enquêtés qui y assistent mais aussi, à l'inverse, à travers ceux qui s'en absentent. La réunion, au contraire du transfert, est évitable si on le souhaite. Tous les arrivants ne sont pas présents et s'absentent majoritairement ceux qui estiment connaître suffisamment Paris ou valorisent une autonomie d'action pour pouvoir se passer d'un tel moment.

\section{Une réception différenciée pour ceux qui reviennent}

Les enquêtés «revenants» ont des comportements contrastés vis-à-vis de cette réunion et témoignent de la différence des investissements selon les configurations de voyage. Il y a une partition entre ceux qui reviennent pour une seconde ou une troisième visite et qui assistent à la réunion mais dans une attitude dilettante et distancée et, enfin, ceux qui affichent un nombre plus important de visites (cinq fois et plus) et ne s'y rendent pas, car l'autonomie est un principe fort de leurs conduites dans la ville. Pour les premiers, il s'agit d'être présent pour la convivialité du moment, temps propice à l'interconnaissance. En conséquence, la généralité des informations 
qui sont fournies, parce qu'elles ressortent du domaine du connu, entraîne une attitude globale d'inattention : «Bon, je dois dire que je n'ai pas écouté si attentivement parce que c'est toujours très général, comment aller à tel endroit et... Elle donne toujours des conseils sur comment utiliser la carte et les habitudes du métro et des choses comme ça, donc je le savais déjà et j'ai un peu "zappé" ( (Paavo, 43 ans, 2006 : communications personnelles). Ceux dont le contact avec Paris est familier justifient leur absence par une valorisation prononcée de l'autonomie. Le rejet des préconisations extérieures est net, et la place accordée dans le processus de décision est ici centrale et non négociable :

Je ne suis jamais allé dans ces réunions d'informations, enfin si, la première fois, je crois, mais j'aime bien faire les choses seul. Je déteste cette sensation de m'asseoir et d'entendre le guide : "il y a ça à faire, et ça à faire", je n'aime pas ça. J'aime le faire moi-même et vérifier moimême et trouver moi-même [...] ça ne m'intéresse pas. Mais je comprends les gens qui viennent pour la première fois et les gens qui ne voyagent pas beaucoup, bien sûr, c'est bien pour eux (Christian, 53 ans, 2006 : communications personnelles).

Au final, l'effet opératoire de cette réunion est, avant tout, la recherche d'une optimisation du voyage pour les touristes les plus désireux et demandeurs d'un cadrage général. Si d'autres raisons peuvent être avancées (chercher des informations plus spécifiques ou le contact du collectif), il reste que l'analyse du contenu des informations diffusées montre qu'elles opèrent comme une première sélection dans le choix et la retenue d'éléments au sein d'un ensemble varié qui facilite ainsi la programmation en proposant un espace restreint de possibles. La pratique de la ville s'appuie sur ce premier balisage où la représentation des lieux dignes d'intérêt est plus claire, parce que «déjà donnée». Cette réunion d' «introduction» à la ville fonctionne efficacement pour la plupart des enquêtés inscrits dans une posture de découverte initiatique.

\section{Des relations plurielles aux visites guidées} Le guide, passeur compétent pour «lire» la ville

Le guide-interprète qui intervient spécifiquement lors des visites ponctuelles organisées (le Château de Versailles, le musée du Louvre, le «tour de ville» en autocar) constitue pour les enquêtés un intermédiaire savant en mesure d'exposer et de décrire la ville. Trois aspects qui renvoient chacun à différentes facettes de la ville sont dénombrables.

Premièrement, c'est la ville dans sa dimension historique ou esthétique qui est expliquée, où l'on rend compte de la valeur d'un site ou d'une œuvre. C'est autant le rôle du «tour de ville» que les visites à Versailles et au Louvre. Ils permettent le recueil d'un ensemble de connaissances historiques et esthétiques qui ont une fonction de dévoilement qui s'illustre moins dans la révélation d'éléments potentiellement cachés que dans l'éclairage de sites redevables du passé, notamment les formes urbaines caractéristiques de la ville historique. Cependant, c'est surtout la divulgation concrète, in vivo, d'un discours qui complète l'activité visuelle et qui facilite l'incarnation d'une représentation originelle — généralement assez abstraite — dans des formes et des sites précis.
Deuxièmement, ce sont des particularités de la ville, dans ses détails et ses originalités. C'est une lecture plus localisée attachée à faire ressortir la partie plutôt que l'ensemble (l'excursion "tour de ville» est ici davantage concernée). Les discours pointent des curiosités logées dans la ville qui passeraient vraisemblablement inaperçues et qui demandent, en conséquence, un savoir spécifique.

La guide disait des choses très diverses [...] elle donnait des informations très détaillées sur les bâtiments, les monuments. Et c'était très surprenant pour moi, en fait, je pensais qu'elle lisait un papier, parce que quelqu'un de normal ne peut pas vraiment en savoir autant sur toutes ces choses. J'ai eu l'impression qu'elle lisait parce que je ne peux pas imaginer que quelqu'un sache autant de petits détails sur tous ces bâtiments et tous ces endroits! (Gabriel, 56 ans, 2006 : communications personnelles).

Ce sont des détails significatifs pour la culture nationale (l'hôtel où vécut Sibélius), des lieux comme des boutiques de luxe spécialisées (dans la maroquinerie et la chapellerie) qui sont l'expression d'une différence (car réservée à une élite économique) et qui surprennent. Ce tableau hétéroclite met la lumière sur la diversité des ressources attractives de la métropole. Le discours du guide extrait de la vie urbaine moult curiosités possibles qui nourrissent un paradoxe en produisant la sensation de la pluralité et du divers à partir d'un lieu ou d'un objet spécifique. Situant la focale à l'échelle de la rue, dans la sélection d'un commerce étonnant ou d'un bâti (la plus petite maison de Paris, par exemple), c'est le sentiment de l'immensité de la "grande ville» qui ressort. En définitive, c'est la mise en forme d'une représentation à dominante « anecdotique» de la ville, comme regard du particulier sur les composantes urbaines.

Enfin, c'est la ville dans sa dimension actuelle et vivante qui est soulignée. Le guide tente de donner forme à un espace qui ne soit pas figé que dans le bâti et l'histoire, ni dans la multiplicité des potentialités de la grande ville. Dans cette optique, le guide aborde la ville sous divers angles : l'aspect foncier et la cherté des loyers, les modes de vie et l'importance des cafés comme lieux de vie sociale, permettant de tisser des comparaisons au sein desquelles le touriste se positionne. "Elle [la guide] nous a dit que les Français passaient beaucoup de temps dans les cafés, que c'était comme une deuxième maison. Nous, on a plus de grands appartements, donc nous passons plus de temps dedans», explique Teiva, 51 ans (2006 : communications personnelles). La politique est également évoquée. Le croisement de ces regards permet de se représenter l'actualité de la vie parisienne.

C'est ainsi une triple partition entre un regard sur les aspects «monumentaux» de la ville, mais aussi ses dimensions «banales» (les singularités subjectivement perçues dans l'environnement urbain sans qu'elles soient associées à un aspect à priori touristique). C'est également la ville «contemporaine», dans son actualité et ses événements. Le guide apparaît comme un "passeur compétent» par sa capacité à bien juger et à transmettre, ouvrant ainsi à d'autres aspects de la représentation. Cependant, tous les enquêtés ne sont néanmoins pas désireux d'une telle lecture tierce. 
Les attitudes de distance face aux excursions

En effet, la distance aux excursions se lit dans plusieurs attitudes qui sont à la fois conjoncturelles (liées aux conditions de la visite ou à des changements dans le cours du voyage) et structurelles (davantage déterminées par l'aspect financier ou l'acculturation à Paris).

La conjoncture du voyage introduit parfois des imprévus qui rendent difficile, voire impossible la tenue des excursions. Outre les facteurs extérieurs (une grève, des travaux, etc.), il y a des imprévus imputables aux touristes eux-mêmes. Par exemple, c'est le sentiment d'avoir dépensé, dans les premiers jours, plus que la somme globalement budgétée et on préfère alors se restreindre sur une excursion programmée. Ce sont également les conditions résultant de la visite qui, en obturant le plaisir, produisent une distance par une attitude de dépréciation à postériori. Ces réactions restent rares, mais on repère néanmoins des déceptions liées à la rapidité et à la promiscuité humaine dans lesquelles certaines visites se réalisent, et qui font des excursions au château de Versailles et au musée du Louvre un parcours soumis à une temporalité rigide et à une perméabilité à la fois sonore et physique visà-vis des autres groupes de visiteurs. La répartition initiale de l'attention entre l'activité visuelle et auditive (dirigées vers les œuvres et le guide) est malmenée par l'environnement qui demande d'ajuster sa position pour suivre la visite. Un touriste explique :

Nous sommes allés à Versailles. En fait... c'est un très bel endroit mais j'ai détesté parce qu'il y a trop de touristes là-bas. Je veux dire... il faisait si chaud et les bâtiments, le château, et tu vas... je voulais juste prendre quelques photos mais il y a trop de touristes dans un seul endroit... on est dans un endroit magnifique, on veut prendre des belles photos et on nous dit : "nous partons maintenant, allez-y, il y a un autre groupe qui arrive..." (Heidi, 40 ans, 2006 : communications personnelles).

Au final, un tel environnement limité dans le temps et producteur d'interférences amoindrit la qualité de la visite.

$\mathrm{Au}$ niveau structurel, la dimension explicative la plus prégnante de la distanciation aux excursions réside dans la valorisation de l'autonomie. Elle illustre la recherche de pratiques urbaines librement décidées et gérées «individuellement», sans que l'itinéraire soit préalablement tracé et une temporalité fixée. Toutefois, ce comportement est redevable de deux types de configurations : l'une qui ressort de touristes « revenants » ayant une attitude homogène de rejet; l'autre est celle des touristes "primo arrivants» qui ne rejettent que les excursions qu'ils jugent attentatoires à l'autonomie :

[On a fait] juste le Louvre et Versailles mais le reste, nous voulions voir par nous-mêmes, nous voulions avoir la liberté de choisir, d'aller où nous voulions, et pas dans ces trucs organisés [...]. C'est parce que nous voulions faire notre propre emploi du temps... donc nous n'aimons pas faire des choses en groupe, nous voulons voir les choses de plus près... comme ça nous avons le sentiment de choisir les endroits où nous allons $[\ldots]$ nous voulons avoir notre propre point de vue, et prendre notre temps... ce n'est pas possible avec un groupe... le guide s'en va et nous devons suivre le groupe et... nous ne pouvons pas regarder de plus près ce que nous voulons. Donc nous y allons seules, comme ça nous pouvons prendre notre temps... et aller où on veut [...] nous ne voulons pas suivre un emploi du temps qui a été fait par d'autres... (Brigitta, 44 ans, 2006 : comunications personnelles).

Ainsi, le «tour de ville» est facilement rapporté à une visite touristique classique qui ne fait qu' «expliquer» la ville à grands traits, portée par l'effet «autocar» et qui peut être rédhibitoire. Pour les visites plus valorisées, elles sont davantage saisies comme des excursions au caractère historique et artistique, donc portées par une finalité de connaissances. Ces visites appellent la présence d'un guide comme tierce personne spécialisée dans ces domaines spécifiques (l'art, l'histoire). Il y a donc, dans l'esprit des touristes qui ne choisissent que ces excursions, le partage entre un tourisme «de visite», dans son expression basique et sommaire (voir la ville), peu valorisante, et un tourisme plus «culturel» lié aux lieux d'Histoire, jugés plus positivement. Pour les touristes qui reviennent à Paris et ne participent à aucune des excursions proposées, c'est moins un principe général que le désaccord avec ces visites, jugées trop classiques.

En résumé, trois logiques sont distinguables pour saisir la relation aux excursions proposées : il y a celle de l'acceptation où se mêlent néanmoins une appréciation des visites selon les finalités visées (connaissance pratique ou culturelle), une distance justifiée par la désadéquation entre l'offre et la demande (les visites sont jugées trop classiques) et un rejet explicite où l'hétéronomie pose problème (un groupe, des horaires, etc.). Cette triple partition «acceptation/distance/rejet» suit globalement l'expérience parisienne acquise par le nombre de visites : plus elles sont importantes, moins les offres proposées semblent correspondre aux désirs des touristes.

\section{Conclusion : la ville comme texte à déchiffrer?}

Les relations entre les touristes et la structure d'encadrement interrogent directement la manière dont le voyage est « rationalisé» à travers un ensemble de moyens et de ressources. La logique du voyagiste traduit assez nettement une offre de sécurisation en filtrant l'apparente complexité de la ville par nombre d'informations qui indiquent et signalent des lieux dignes d'intérêts pour visiter, se promener, acheter, etc. Cette offre est également lisible sous l'angle de l'efficacité où il s'agit de produire des effets d'ajustement et d'adéquation pour pallier les différences avec le territoire et son fonctionnement. Cette diffusion d'information censée rassurer et faciliter les engagements dans la ville est néanmoins diversement appropriée. C'est précisément parce que ces moments sont pensés et développés pour offrir une stabilité face à un environnement aléatoirement maîtrisé que les enquêtés novices répondent favorablement et que ceux qui estiment disposer de ressources suffisantes y participent dans une écoute partielle ou s'en éloignent. Pour les moins initiés, la ville apparaît comme un espace plus ou moins obscur, tel un texte à déchiffrer. L'analogie n'est pas nouvelle — Barthes (1970) soulignait déjà la dimension idéogrammatique de la ville et son lien possible avec l'univers textuel — et trouve sa pertinence dans la 
réalité complexe de l'urbain, demandant à être interprété pour pouvoir en comprendre la structure. Urbain (1991) a souligné cette nécessité du déchiffrement par le caractère équivoque des villes aujourd'hui : «mosaïque de territoires imbriqués difficilement déchiffrables» (Urbain, $1991: 183$ ).

La pluralité intrinsèque de la ville et son caractère imbriqué et stratifié par le temps renforcent la recherche d'intelligibilité et le souci d'une interprétation pour la rendre compréhensible. Le recours à un encadrement permet de répondre à un questionnement basique qui interpelle le visiteur confronté à l'orientation et à la découverte : comment cela fonctionnet-il, quels sont les lieux intéressants, etc.? Urbain (2002) pose pourtant la question de l'intelligibilité en rapport avec l'existence du panorama qui éclaircit l'environnement que l'on découvre, ouvrant à une vue d'ensemble de la ville, le texte sous les yeux et l'interprétation à portée de soi. Dans la situation exposée ici, c'est moins le panorama que les visites et les réunions qui servent de lecture en facilitant une intelligibilité des lieux. Le voyagiste a ainsi une fonction similaire au panorama où la ville s'éclaircit progressivement en fonction des discours des encadrants et de l'avancée physique dans la ville. Les encadrants seraient alors comme des lecteurs avertis qui, à défaut de proposer aux touristes une lecture intégrale (trop chronophage), choisiraient des passages et citeraient ceux qui sont dignes d'intérêt (lecture «historique»), n'oubliant pas de petits faits non essentiels mais pittoresques (lecture «anecdotique») et la réinscription de l'«espace-livre» dans une perspective contemporaine (lecture «actuelle»).

\section{Références}

APUR (2001) Le tourisme de groupe à Paris. Éléments de diagnostic, Paris : Atelier Parisien d'Urbanisme. 41 p.

BARTHES, Roland (1970) L'empire des signes, Paris : Skira. 157 p.

BAUER, Ana (2007) «Le tourisme de groupe n'est pas mort», Cahier Espaces, $\mathrm{n}^{\circ} 95$, p. 16-17.

BELLEFLEUR, Michel (2002) Le loisir contemporain. Essai de philosophie sociale, Sainte-Foy : Presses de l'Université du Québec. 192 p.

BIRGITTA (2006) touriste, 44 ans, entrevue réalisée à Paris, le 5 avril.

BOORSTIN, Daniel J. (1963) L'image ou ce qu'il advint du rêve américain, Paris : Julliard. 325 p.

BOWMAN, Glenn (1992) «The Politics of Tour Guiding : Israeli and Palestinian Guides in Israel and the Occupied Territories », DANS HARRISON, David (sous la direction de), Tourism and the Less Developed Countries, p. 121-134. Londres : Belhaven.

BOYER, Marc (1999) Histoire du tourisme de masse, Paris : Presses Univeristaires de France. 128 p.

CHAUVEL, Louis (2006) Les classes moyennes à la dérive, Paris : Seuil. 108 p. CHRISTIAN (2006) touriste, 53 ans, entrevue réalisée à Paris, le 15 mai.

COHEN, Erik (1972) «Toward a Sociology of International Tourism», Social Research, vol. 39, p. 164-189.

COHEN, Erik (1973) «Nomads from Affluence: Notes on the Phenomenon of Drifter Tourism ", International Journal of Comparative Sociology, vol. 14, p. 89-103.

COHEN, Erik (1985) «The Tourist Guide: The Origins, Structure and Dynamics of a Role», Annals of Tourism Research, vol. 12, p. 5-29.
COLLIOT-THÉLÈNE, Catherine (2006) Sociologie de Max Weber, Paris : La Découverte. $122 \mathrm{p}$.

DALHES, Heidi (2002) «The Politics of Tour Guiding. Image Management in Indonesia ", Annals of Tourism Research, vol. 29, n 3, p. 783-800.

DUHAMEL, Philippe et Rémy KNAFOU (2007) «Le tourisme dans la centralité parisienne», DANS SAINT-JULIEN, T. et R. Le Goix (éditeurs) La métropole parisienne. Centralités, inégalités, proximités, p. 39-64. Paris : Belin.

ENZENSBERGER, Hans-Magnus (1962, rééd.1973) Culture ou mise en condition? Paris : Les Lettres Nouvelles. 440 p.

FAGNONI, Edith et Colette AYMARD (2002) «Entre inertie et dynamisme touristique. Le cas parisien ", Téoros, vol. 21, n 1 , p. 4-11.

GABRIEL (2006) touriste, 56 ans, entrevue réalisée à Paris, le 21 mars. HEIDI (2006) touriste, 40 ans, entrevue réalisée à Paris, le 5 février. HOLLOWAY, Christopher (1981) «The Guided Tour. A Sociological Approach ", Annals of Tourism Research, vol. 8, p. 377-402.

HOLM, Olaf (2003) «De Paris à Berlin, de Bruxelles à New York. Visiter "autrement" les villes du monde», Cahier Espaces, n 78, p. 134-143.

KHARAS, Homi (2010) «The emerging middle class in developping countries», Working Paper no 85, OECD Development Centre.

LAHIRE, Bernard (2005) L'esprit sociologique, Paris : La Découverte. 271 p.

LANQUAR, Robert (1995) Agences et industrie du voyage, Paris : Presses Universitaires de France. 127 p.

LI, Yang (2000) «Geographical Consciousness and Tourism Experience», Annals of Tourism Research, vol. 27, n 4, p. 863-883.

MACCANNELL, Dean (1976, rééd.1989) The tourist. A new theory of the leisure class, New York : Schocken Books. 214 p.

NOORA (2006) touriste, 55 ans, entrevue réalisée à Paris, le 16 mai.

OTCP (2012) «Le tourisme à Paris. Chiffres clés 2011 », Office de Tourisme et de Congrès de Paris. $40 \mathrm{p}$.

PAAVO (2006) touriste, 43 ans, entrevue réalisée à Paris, le 25 mars. PAULIINA (2006) agent réceptif, entrevue réalisée à Paris, le 7 avril. POND, Kathleen (1993) The Professionnal Guide: Dynamics of a Tour Guiding, New York : Van Nostrand Reinhold. 250 p.

RISTO (2006) touriste, 56 ans, entrevue réalisée à Paris, le 21 avril.

SALLET-LAVOREL, Hélène (2003) «Encourager le rapprochement entre visiteurs et Franciliens. Pour un tourisme urbain participatif en Ile-deFrance», Cahiers Espaces, n' ${ }^{\circ}$ 78, p. 118-133.

SCHMIDT, Catherine (1979) «The Guided Tour : Insulated Adventure. Urban Life: A Journal of Ethnographic Research ", Journal of Contemporary Ethnography, vol. 8, p. 441-468.

TEIVA (2006) touriste, 51 ans, entrevue réalisée à Paris, le 25 avril. TEUVO (2006) touriste, 44 ans, entrevue réalisée à Paris, le 2 mai.

URBAIN, Jean-Didier (1991, rééd. 2002) L'idiot du voyage. Histoires de touristes, Paris: Payot et Rivages. 353 p.

URBAIN, Jean-Didier (2003) Ethnologue, mais pas trop, Paris : Payot et Rivages. 285 p.

VENETO, Joe (2007) «Les nouveaux marchés du tourisme de groupe», Cahier Espaces, no 95, p. 10-15.

WEBER, Max (1921, rééd. 1971) Économie et Société, Paris : Plon. 410 p.

WICKENS, Eugenia (2002) «The Sacred and the Profane. A Tourist Typology", Annals of the Tourism Research, vol. 29, n 3, p. 834-851. 